DEMOGRAPHIC RESEARCH

VOLUME 45, ARTICLE 33, PAGES 1011-1040

PUBLISHED 4 NOVEMBER 2021

https://www.demographic-research.org/Volumes/Vol45/33/

DOI: 10.4054/DemRes.2021.45.33

Research Article

A world apart: Levels and determinants of excess mortality due to COVID-19 in care homes: The case of the Belgian region of Wallonia during the spring 2020 wave

Olivier J. Hardy

Dominique Dubourg

Mélanie Bourguignon

Simon Dellicour

Thierry Eggerickx
Marius Gilbert

Jean-Paul Sanderson

Aline Scohy

Eline Vandael

Jean-Michel Decroly

(C) 2021 Olivier J. Hardy et al.

This open-access work is published under the terms of the Creative Commons Attribution 3.0 Germany (CC BY 3.0 DE), which permits use, reproduction, and distribution in any medium, provided the original author(s) and source are given credit.

See https://creativecommons.org/licenses/by/3.0/de/legalcode. 


\section{Contents}

1 Introduction 1012

$2 \quad$ Materials and methods 1014

2.1 Area of interest and institutional context related to care homes 1014

2.2 COVID-19 mortality data 1015

2.3 All-cause annual mortality data in $2017 \quad 1016$

$\begin{array}{lll}2.4 & \text { Analytical strategy } & 1017\end{array}$

3 Results 1019

3.1 Distribution of COVID-19 death rate per institution 1020

3.2 Temporal dynamics of COVID-19 deaths inside and outside FE 1021

3.3 Age and sex structure effect on COVID-19 death rate 1022

3.4 COVID-19 death rates by sex and age group - health condition effect 1024

3.5 All-cause annual death rate by sex and age group - a proxy for overall 1025

3.6 Comparison of COVID-19 and annual ASDR to infer differences in 1026 contamination rates between populations

4 Discussion $\quad 1027$

5 Conclusion 1031

6 Acknowledgments 1031

$\begin{array}{ll}\text { References } & 1032\end{array}$

$\begin{array}{ll}\text { Appendix } & 1038\end{array}$ 


\title{
A world apart: Levels and determinants of excess mortality due to COVID-19 in care homes: The case of the Belgian region of Wallonia during the spring 2020 wave
}

\author{
Olivier J. Hardy ${ }^{1}$ \\ Dominique Dubourg ${ }^{2}$ \\ Mélanie Bourguignon ${ }^{3}$ \\ Simon Dellicour ${ }^{4}$ \\ Thierry Eggerickx ${ }^{3}$
}

\author{
Marius Gilbert ${ }^{4}$ \\ Jean-Paul Sanderson ${ }^{3}$ \\ Aline Scohy ${ }^{5}$ \\ Eline Vandael ${ }^{5}$ \\ Jean-Michel Decroly ${ }^{4}$
}

\begin{abstract}

\section{BACKGROUND}

In Western countries, COVID-19 has been particularly deadly for care home residents.

\section{OBJECTIVE}

To understand the role of age and sex structures, health frailty, and contamination dynamics in COVID-19 mortality in populations living inside and outside care homes.
\end{abstract}

\section{METHODS}

We compared COVID-19 death data recorded in March-June 2020 in Wallonia (southern Belgium) for populations living inside and outside care homes, using annual death data (all-cause mortality in 2017) to assess the health condition of each population.

\section{RESULTS}

Sixty-four percent of COVID-19 deaths were residents in care homes, where the outbreak started after that in the external population, but at a faster pace. The death rate varied between $0 \%$ and $340 \%$ (mean $43 \%$ ) per care home, increasing with the number of both residents and staff. All-cause and COVID-19 mortality rates increased exponentially with age but were much higher in care homes. The ratio of male $(\mathrm{M})$ to female $(\mathrm{F})$ death rates was 1.6 for all-cause mortality and 2.0 for COVID-19 mortality (both confirmed and

\footnotetext{
${ }^{1}$ Université Libre de Bruxelles, Belgium. Email: ohardy@ulb.ac.be.

${ }^{2}$ Agence pour une Vie de Qualité, Belgium.

${ }^{3}$ Université catholique de Louvain, Belgium.

${ }^{4}$ Université libre de Bruxelles, Belgium.

${ }^{5}$ Sciensano, Belgium.
} 
suspected). The COVID-19 mortality reached 24\% (M) and 18\% (F) of the all-cause mortality rate in care homes, compared to 5\% (M) and 4\% (F) outside care homes.

\section{CONCLUSIONS}

The COVID-19 mortality rate was 130x higher inside than outside care homes, due to the near multiplicative effects of differences in the residents' age and sex structure (11x), health frailty (3.8x), and infection risk (probably $3.5 \mathrm{x}$ ).

\section{CONTRIBUTION}

Care homes should be treated as a very specific population in epidemiological studies due to their extreme vulnerability to COVID-19.

\section{Introduction}

Since its appearance in China at the end of 2019, the COVID-19 pandemic has been known to cause higher mortality among older people and in males rather than females (Bauer et al. 2021; Dudel et al. 2020; Medford and Trias-Llimós 2020; Wenham, Smith, and Morgan 2020), as well as in people suffering from various comorbidity factors (Nepomuceno et al. 2020). In Europe and North America, COVID-19 has had a dramatic impact on people living in care homes (Comas-Herrera et al. 2020; ECDC 2020; Fisman et al. 2020; Ladhani et al. 2020; Petretto and Pili 2020), even though restricting family visits was one of the first policies applied to contain the outbreak (Comas-Herrera, Ashcroft, and Lorenz-Dant 2020; Verbeek et al. 2020). The high incidence of COVID19 in care homes is likely related to everyday contact with the nursing staff facilitating contagion (Arons et al. 2020; Ladhani et al. 2020), especially as many countries failed to provide sufficient personal protective equipment (PPE) for care homes at the beginning of the pandemic, usually saving protective equipment in limited supply (e.g., chirurgical masks and gloves) for hospitals (Logar 2020 for Italy, Rada 2020 for Spain, Quigley et al. 2020 for the United States, Szczerbińska 2020 for an international comparison). However, to assess if the COVID-19 mortality rate was higher in care homes it is necessary to factor out age and sex effects, as they are mostly populated by old people and women are over-represented in care homes (Einiö et al. 2012). It is also important to take into account the health frailty of the population living in care homes (Falconer and O'Neill 2007), since people often enter care homes due to deteriorating health status (Herm, Poulain, and Anson 2014), and that care home residents tend to have a lower social status than the rest of the population (Laferrère et al. 2013). Finally, distinguishing different categories of care home according to their size and the type of health assistance they provide could be instructive when comparing how COVID-19 has affected 
populations that differ by their general health frailty and/or by the risk of contamination through nursing services.

As PCR testing capacities were also very limited at the beginning of the outbreak there was often a lack of testing to assess whether deaths occurring within care homes resulted from COVID-19. In some countries, like Belgium, the reporting of COVID-19 deaths by national authorities has included people suspected of having died from COVID19 due to characteristic symptoms, despite the absence of PCR testing (Comas-Herrera et al. 2020). The excess of deaths in spring 2020 compared to previous years according to national register data clearly supported this inclusive approach (Sciensano 2020; Bustos Sierra et al. 2020). From 8 March to 9 May 2020, during the most intense phase of the epidemic in terms of mortality, 8,735 COVID-19 deaths (confirmed and suspected) were recorded in Belgium, while over the same period an excess mortality of 8,280 deaths was recorded in the National Register compared to the average mortality of 2015-2019 (a difference of 455 deaths, barely 5\%). However, it has been suggested that social isolation and the stress caused by anxiety could strongly affect elderly people during lockdown (Armitage and Nellums 2020) and might also have been responsible for a substantial portion of excess mortality in nursing homes (Trabucchi and De Leo 2020). Although it is extremely hard to retrospectively identify the cause of death, we can expect that if the COVID-19 mortality rate is affected by age and sex following a particular pattern, when the diagnosis is correct, suspected COVID-19 deaths should display the same pattern as confirmed COVID-19 deaths.

Our objective is to characterize mortality resulting from COVID-19 in two different populations: residents of care homes for elderly people and the rest of the population, while accounting for age and sex effects as well as the general health status of each population. We focus on the southern Belgium region of Wallonia because we have good quality data on both COVID-19 deaths and all-cause annual deaths in care homes. First, we show the overall difference in COVID-19 mortality between populations living in and out of care homes, as well as the mortality variation between care homes according to the importance of medical assistance (distinguishing nursing homes from residential homes) and their size. We also compare the temporal dynamics of mortality in and out of care homes because we hypothesize (1) that the outbreak in care homes started later than outside, and (2) that the potentially high contagion within care homes might have caused a more rapid spread of the virus. To ensure that our results are not biased by strong geographic patterns we assess the spatial autocorrelation of mortality rate per care home. Second, we seek to explain the mortality differential within and outside care homes through two factors: differences in structure by age and sex (structure effect) and differences in COVID-19 age-and-sex-specific death (health condition effect). We verify whether the age effect follows the Gompertz exponential law, which can reflect vulnerability to external causes (Ricklefs and Scheuerlein 2002), and whether the sex 
effect deviates from that observed for annual mortality. We attempt to explain the health condition effect, which results from (1) the unequal incidence of the disease in the populations being compared (infection effect), and (2) the greater or lesser vulnerability of individuals once they have contracted SARS-CoV2 (frailty effect). The latter can be approximated by the usual level of mortality outside the COVID-19 health crisis in the populations studied. In the absence of systematic testing it is not possible to measure the unequal incidence of the disease in the compared populations. However, we show that it is possible to estimate it by comparing mortality caused by COVID-19 and all-cause annual mortality, because the two are tightly correlated in each population. Through these different analyses we provide a better understanding of the effect of key factors at the origin of the high incidence of COVID-19 on the mortality in care homes.

\section{Materials and methods}

\subsection{Area of interest and institutional context related to care homes}

Due to data availability of both COVID-19 and all-cause annual deaths, we focus on the French-speaking part of Wallonia in southern Belgium (i.e., the Walloon Region without the municipalities belonging to the German-speaking community of eastern Wallonia). In this area, comprising c. 3.6 million inhabitants, all residential facilities for elderly people $(\mathrm{FE})^{6}$ are supervised by the regional administration AViQ (Agence pour une Vie de Qualité, https://www.aviq.be), which collected statistics regarding COVID-19 deaths and performed recurrent systematic surveys on yearly mortality, the last one occurring in 2017. For some analyses, in the FE category we distinguish homes for elderly people (HEP), which host essentially old people who require assistance with daily meals, housekeeping, and/or daily toilet, but do not require substantial health care, and nursing homes (NH), which essentially host old people with the same needs as HEP residents but also people requiring additional health care due to more or less severe pathologies. According to the standards established by the Code Réglementaire Wallon de l'Action Sociale et de la Santé, the number of nursing staff per 30 residents is 4.5 in HEP and 12.1 in $\mathrm{NH}$. The latter must also have at least five nurses, one coordinating doctor, and 0.1 full-time equivalent specialists in palliative care. $\mathrm{NH}$ also have written procedures for hand hygiene and the isolation of residents with an infection that carries a contamination risk. On average, approximately half of the $\mathrm{NH}$ bed capacity is devoted to residents requiring healthcare services. In this paper, FE residents will be referred to as the FE

\footnotetext{
${ }^{6}$ The terms used here to designate different categories of care homes are those indicated on the Healthy Belgium website of the Belgian federal government (https://www.healthybelgium.be/en/health-system-performanceassessment/specific-domains/care-for-the-elderly)
} 
population and the rest of the population living in the French-speaking part of Wallonia as the OUT-FE population.

\subsection{COVID-19 mortality data}

Our analysis of mortality by COVID-19 in and out of FE covers the period between March 13 and June 30, 2020. Our data came from two sources. First, AViQ collected death data attributed to COVID-19 as reported by all 573 FE (446 NH and 127 HEP) existing in 2020 (including death date, age, and sex). These deaths occurred either in hospitals (28\% of cases), in which case a PCR and/or scanner test confirmed the COVID19 diagnostic, or in NH or HEP institutions ( $72 \%$ of cases) where tests were available for only $27 \%$ of cases, so the majority of these cases were suspected to be the result of COVID-19 following the diagnosis of the attending physician and given the presence of characteristic symptoms (upper or lower respiratory tract infection, fever or chills, cough, shortness of breath or difficulty breathing, headache, new loss of taste or smell, etc.; Peeters et al. 2021). Second, Sciensano, the Federal Institution responsible for monitoring health in Belgium (https://www.sciensano.be), collected COVID-19-related death data reported by hospitals and other settings, such as the agencies monitoring care homes (including AViQ). We extracted from their dataset all COVID-19-related deaths (suspected and confirmed) reported in Wallonia (excluding the German-speaking municipalities). To distribute deaths among Belgian regions the Sciensano dataset considers the place of death rather than the place of residence. However, according to partial data on the place of residence of deaths, less than $1 \%$ of deaths occurring in the Brussels or Walloon regions concerned people residing in another region, so we overlooked this source of bias.

To assess the heterogeneity of COVID-19 mortality among institutions, the crude COVID-19 death rate per institution was computed by dividing the number of reported deaths by the total bed capacity of the institution. This ratio underestimates the actual mortality rate but should remain realistic given that $>90 \%$ of the bed capacity was usually occupied at the beginning of the outbreak. We considered an institution to be a physical site with a particular approval number in the AViQ database, although some sites are managed by the same administrative entity. We tested if there was a spatial pattern in mortality by computing Moran's I statistic on crude death rate per FE institution, using binary weight matrices for institutions separated by the distance intervals $0-10,10-20$, 20-50, 50-100, and $>100 \mathrm{~km}$.

To characterize the temporal dynamics of COVID-19 deaths from March 13 until June 30, 2020, we computed the cumulated number of deaths for FE residents and for the OUT-FE population (by subtracting the AViQ data from the Sciensano data for 
Wallonia). To assess the delay and pace of the outbreaks that occurred in each population, we also computed the median date at which people from each population died (i.e., when $50 \%$ of all deaths occurred) as well as the dates corresponding to the 0.05 and 0.95 quantiles.

To assess the age and sex effects, COVID-19 Age-Specific Death Rates (ASDR) were computed per sex and 5-year age classes, separately for the FE and OUT-FE populations, as the ratio of the number of COVID-19 deaths to the corresponding ageand sex-specific population sizes estimated on January 1, 2020 (Appendix). For FE, these population sizes were derived from the population survey established on January 1, 2018 by AViQ (see below). This survey provides more reliable data on the FE population than the data on collective households ${ }^{7}$ from the National Population Register because some residents in FE remain domiciled in their former place of residence, which leads to an underestimation of the FE population by the National Register. To estimate the population by age in FE on January 1, 2020, a multiplication factor reflecting the change in total bed capacity between January 1, 2018, and January 1, 2020 was applied to the data for January 1, 2018 (factor of 42103/40852 $=1.031$ for NH and 6249/6865 $=0.910$ for HEP). This estimate may seem potentially biased, since it keeps the age structure of the FE population constant and thus does not take into account possible cohort effects (e.g., the cohorts born in the early 1940s are smaller than the cohorts born in the late 1930s or late 1940s). However, it seems preferable to other solutions such as assuming that the FE population evolves in a similar way to the whole population. Moreover, the selected solution does not alter the results. Indeed, National Population Register data on people 65 and over living in collective households (composed mainly of people living in FE) show that their age structure remained almost constant between 2018 and 2020: the proportion of each age and sex group among those 65 and over is approximately the same on both dates (maximum difference between proportions is 1.3 percentage points). For the OUT-FE population we used the StatBel data (https://statbel.fgov.be), based on the National Register, which provided the population size disaggregated by age and sex for our focal area on January 1, 2020, and subtracted the FE population.

\subsection{All-cause annual mortality data in $\mathbf{2 0 1 7}$}

We compared the overall health frailty of each population using their respective ASDR before the COVID-19 epidemic. AViQ conducted a survey in 2017 asking each of the $587 \mathrm{FE}$ (440 NH and $147 \mathrm{HEP}$ ) existing that year to report deaths (including birth and death dates) throughout the year, and to report their population on January 1, 2018. Here,

\footnotetext{
${ }^{7}$ In Belgium, collective households are groups of more than two people living at the same address. They include the population residing in orphanages, prisons, religious communities, and residences for elderly people.
} 
we consider only deaths and the population of long-term residents, excluding people that registered for revalidation short-stays (typically less than a month). Overall, in terms of bed capacity, $93.3 \%$ of $\mathrm{NH}$ and $84.8 \%$ of HEP provided their population statistics, and 91.3\% and $83.2 \%$ reported their 2017 deaths. Sex and age data were available for at least $98 \%$ of the people in each case. To correct raw numbers, they were divided by the proportion of responding institutions (in bed equivalents) and by the proportion of complete sex and age data, so that incomplete data were redistributed across all ages by sex category.

From StatBel population statistics we extracted the population on January 1, 2018, as well as the number of deaths during 2017, disaggregated by age and sex, for our focal area. From this overall population and death number we subtracted the corresponding estimated numbers for FE (based on the AViQ 2017 survey) to estimate the population and associated deaths of people living outside of care homes, i.e., the OUT-FE population. In total, 37,131 deaths (17,978 men and 19,153 women) were recorded in 2017, $22 \%$ of males (M) and $45 \%$ of females (F) being FE residents. Although the OUTFE population parameters were obtained by subtracting approximated FE population parameters from residuals, bias is not expected given that FE residents constitute a minority of all deaths.

Annual ASDR were then computed for 5-year age categories by sex, separately for each population (OUT-FE and FE, in which we also distinguished NH and HEP), by dividing the number of deaths occurring in 2017 by the population size on January 1 , 2018.

\subsection{Analytical strategy}

The distribution of the COVID-19 death rate per institution was established separately for NH and HEP, and within each type of institution separately for the smallest and largest institutions in terms of bed capacity. The criterion used to distinguish small and large institutions is where approximately half of the residents of a particular institution type (NH or HEP) live. We used Mann-Whitney U tests to assess whether the medians of these distributions differ.

As we expected the FE and OUT-FE populations to differ substantially in their age and sex composition, we established their age pyramids using population data on January 1,2020 , and used standardized mortality rates to compare them. For each population, COVID-19 ASDR per 5-year class $(m)$ were computed. Because the size of the HEP population is relatively small, COVID-19 ASDR often lack sufficient precision, so that we generally report them for FE (i.e., NH + HEP) only, and highlight differences between 
$\mathrm{NH}$ and HEP on overall mortality rates because the two populations have very similar age pyramids.

For many diseases, the mortality-age relationship follows an exponential function known as the Gompertz law (Gompertz 1825; Riggs 1991; Imaizumi 1996). Therefore, COVID-19 ASDR were represented on a log scale against the mid-age (a) of each class, and adjusted on an exponential function restricted to age classes between 65 and 99 years (to avoid classes with too small population sizes): $m=c . \mathrm{e}^{b . a}$, where $b$ and $c$ are the adjusted coefficients. We estimated the number of years leading to a doubling of $m$ as $\ln (2) / b$. We used Fisher distribution to compute $95 \%$ confidence intervals of mortality rates following Ars, Dal, and Poulain (1988) as the interval between $\frac{X}{\left(X+(n-X+1) F_{2(n-X+1), 2 X, 0.975}\right)}$ and $\frac{(X+1) F_{2(X+1), 2(n-X), 0.975}}{\left(n-X+(X-1) F_{2(X+1), 2(n-X), 0.975}\right)}$, where $X$ and $n$ represent the age- and sex-specific death number and population size, respectively, and $F_{\mathrm{d} 1, \mathrm{~d} 2, \alpha}$ represents Fisher's distribution at level $\alpha$ with $\mathrm{d} 1$ and $\mathrm{d} 2$ degrees of freedom. The same procedure was applied to annual mortality rates.

To assess the (age and sex) structure and the health condition effects on the mortality differential by COVID-19 within and outside FE, a multiplicative decomposition of the Crude COVID-19 Death Rate (CDR) ratio was applied (Miettinen 1972; Keiding and Clayton 2014; see Appendix). According to this method, the ratio of the CDR within FE to the CDR outside FE is the product of the indirect Standardized Mortality Ratio in FE (SMR_FE) and a confounding risk ratio (CRR). The first is obtained by dividing the CDR within FE by the expected CDR in FE if its population had experienced the COVID-19 ASDR of the population outside FE (ExpCDR_FE). It can be interpreted as a factor that reflects differences in health condition (frailty and prevalence of SARS-COV-2) between populations living in and outside of care homes. The confounding risk ratio (CRR) is obtained by dividing the ExpCDR_FE by the CDR observed outside FE. It measures the effect of differences in age and sex structure on the crude rate ratio. The relative frailty (RF) of the FE population was quantified by the indirect Standardized Mortality Ratio in FE applied to annual death rates (Appendix). Finally, to attempt to factor out the effect of the general health condition of a particular population on COVID-19 mortality, we divided COVID-19 ASDR by the respective annual death rates. We then compared these ratios among populations to tentatively interpret them in terms of relative COVID-19 infection rates (RI). 


\section{Results}

In the French-speaking part of Wallonia, between March 13 and June 30, 2020, COVID19 caused a total of 2,126 deaths in FE (2010 in NH, 116 in HEP) and 1,195 deaths in the OUT-FE population (Table 1). Hence, $64 \%$ of the suspected and confirmed deaths of COVID-19 occurred in FE, even though the population living in these institutions constitutes barely $1.3 \%$ of the Walloon population. Consequently, the crude COVID-19 death rates present an extreme contrast: $44.2 \%$ in FE as compared to $0.34 \%$ outside FE, i.e., a ratio of 130:1.

Table 1: Descriptive statistics of the COVID-19 outbreak in Wallonia (excluding the German-speaking municipalities) in residential facilities for elderly people and in the rest of the population

(a) Population January 1, 2020

\begin{tabular}{|c|c|c|c|c|c|c|c|c|}
\hline & \multicolumn{4}{|l|}{ Total } & \multicolumn{4}{|l|}{$65+$} \\
\hline & \multicolumn{2}{|c|}{ Both sexes } & \multicolumn{2}{|l|}{ Females } & \multicolumn{2}{|c|}{ Both sexes } & \multicolumn{2}{|l|}{ Females } \\
\hline & $\begin{array}{l}x 1,000 \\
\text { persons }\end{array}$ & $\%$ & $\begin{array}{l}x 1,000 \\
\text { persons }\end{array}$ & $\begin{array}{l}\text { \% both sexes } \\
\text { population }\end{array}$ & $\begin{array}{l}x 1,000 \\
\text { persons }\end{array}$ & $\begin{array}{l}\% \text { total } \\
\text { population }\end{array}$ & $\begin{array}{l}x 1,000 \\
\text { persons }\end{array}$ & $\begin{array}{l}\% \text { both sexes } \\
\text { population }\end{array}$ \\
\hline $\mathrm{FE}$ & 48 & 1.3 & 36 & 74.6 & 46 & 95.1 & 35 & 76.2 \\
\hline $\mathrm{NH}$ & 42 & 1.2 & 31 & 74.5 & 40 & 95.2 & 30 & 76.1 \\
\hline HEP & 6 & 0.2 & 5 & 74.7 & 6 & 94.2 & 5 & 76.4 \\
\hline OUT-FE & 3,519 & 98.7 & 1,788 & 50.8 & 626 & 17.8 & 349 & 55.7 \\
\hline Wallonia & 3,567 & 100.0 & 1,824 & 51.1 & 672 & 18.8 & 384 & 57.1 \\
\hline
\end{tabular}

(b) COVID-19 deaths from March 13 to June 30, 2020

\begin{tabular}{|c|c|c|c|c|c|c|c|c|}
\hline & \multicolumn{4}{|l|}{ Total } & \multicolumn{4}{|l|}{$65+$} \\
\hline & Both sexes & & Females & & Both sex & & Females & \\
\hline & persons & $\%$ & persons & $\begin{array}{l}\% \text { of both } \\
\text { sexes deaths }\end{array}$ & persons & $\begin{array}{l}\% \text { of total } \\
\text { deaths }\end{array}$ & persons & $\begin{array}{l}\% \text { of both } \\
\text { sexes deaths }\end{array}$ \\
\hline FE & 2,126 & 64.0 & 1,354 & 63.7 & 2,090 & 98.3 & 1,339 & 64.1 \\
\hline $\mathrm{NH}$ & 2,010 & 60.5 & 1,276 & 63.5 & 1,975 & 98.3 & 1,261 & 63.8 \\
\hline HEP & 116 & 3.5 & 78 & 67.2 & 115 & 99.1 & 78 & 67.8 \\
\hline OUT-FE & 1,195 & 36.0 & 478 & 40.0 & 1001 & 83.8 & 416 & 41.6 \\
\hline Wallonia & 3,321 & 100.0 & 1,832 & 55.2 & 3,091 & 93.1 & 1,755 & 56.8 \\
\hline
\end{tabular}


Table 1: (Continued)

(c) Crude COVID-19 death rates (per 1,000 people)

\begin{tabular}{|c|c|c|c|c|c|c|}
\hline & \multicolumn{3}{|l|}{ Total } & \multicolumn{3}{|l|}{$65+$} \\
\hline & Both sexes & Females & Males & Both sexes & Females & Males \\
\hline $\mathrm{FE}$ & 44.2 & 37.8 & 63.1 & 45.7 & 38.5 & 68.9 \\
\hline $\mathrm{NH}$ & 48.1 & 40.9 & 69.0 & 49.6 & 41.6 & 75.1 \\
\hline HEP & 18.5 & 16.7 & 24.0 & 19.5 & 17.3 & 26.6 \\
\hline OUT-FE & 0.3 & 0.3 & 0.4 & 1.6 & 1.2 & 2.1 \\
\hline Wallonia & 0.9 & 1.0 & 0.9 & 4.6 & 4.6 & 4.6 \\
\hline
\end{tabular}

Notes: Statistics given for the whole population and the population aged 65 and more. $(\mathrm{FE})=$ residential facilities for elderly people, $(\mathrm{NH})=$ nursing homes, HEP $=$ homes for elderly people, OUT-FE $=$ the rest of the population.

Sources: StatBel, AViQ.

\subsection{Distribution of COVID-19 death rate per institution}

Mortality by COVID-19 varied according to the category of FE institution: with 2,010 deaths out of 2,126 (95\%), $\mathrm{NH}$ recorded a crude COVID-19 death rate (48\%) 2.6 times higher than HEP (19\%o) (Table 1). The COVID-19 death rate per institution varied widely between $0 \%$ and $340 \%$ and was affected by both the type of institution and its size (Figure 1). About half of HEP residents lived in small institutions with 14 to 52 beds (mean $=37.8, \mathrm{~N}=84$ ) where $16 \%$ o died from COVID-19, a lower rate than the $21 \%$ o who died in medium-sized HEP institutions with 54 to 148 beds (mean $=74.3, \mathrm{~N}=43$ ). Similarly, about half of NH residents lived in medium-sized institutions with 29 to 104 beds (mean $=76.2, \mathrm{~N}=284$ ) where $40 \%$ o died from COVID-19, a lower rate than the $53 \%$ who died in large NH institutions with 105 to 298 beds (mean $=134.1, \mathrm{~N}=163$ ).

Remarkably, a significant proportion of FE (55\%) recorded no deaths, showing that the effect of the epidemic on care homes was very varied. The proportion of institutions without COVID-19 deaths followed the death rate trend shown above: $81 \%$ and $67 \%$ for small and medium-size HEP institutions, compared to 55\% and 38\% for medium-size and large NH institutions, respectively (Figure 1). Finally, while the crude COVID-19 death rate rarely exceeded $150 \%$ in HEP institutions, it exceeded this threshold in about $10 \%$ of NH institutions, regardless of size (Figure 1). Mann-Whitney U tests on global crude COVID-19 death rates per institution showed that NH and HEP differ significantly ( $\mathrm{z}$-score $=4.67, \mathrm{p}$-value $<0.00001)$ as well as medium $\mathrm{NH}$ versus large $\mathrm{NH}(\mathrm{z}$-score $=-$ 3.07 , $\mathrm{p}$-value $=0.001$ ), while the difference between small and medium HEP ( $\mathrm{z}$-score $=$ $-1.30, \mathrm{p}$-value $=0.097)$ and between medium HEP and medium NH $(\mathrm{z}$-score $=1.20, \mathrm{p}$ value $=0.11$ ) was only marginally significant. 


\section{Figure 1: Cumulated distributions of COVID-19 death rate}

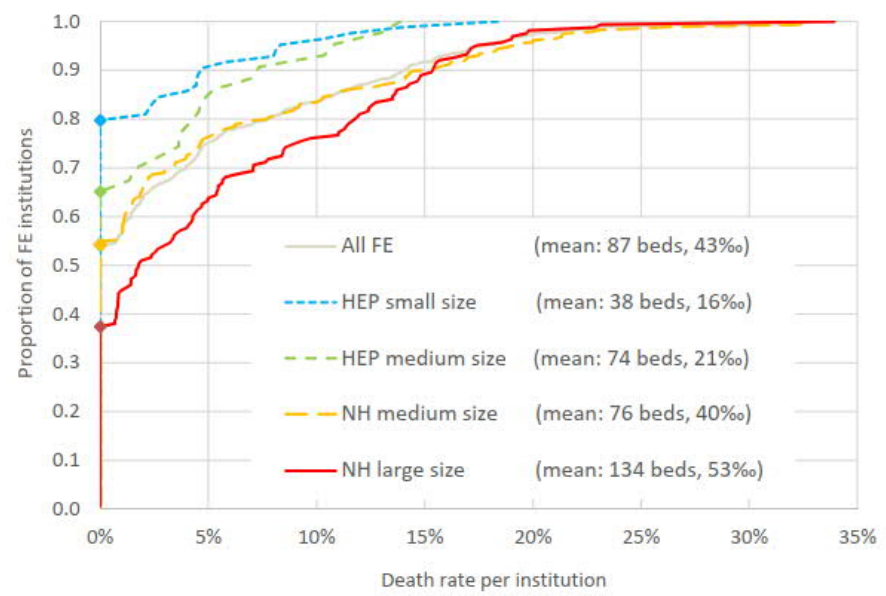

Notes: The symbols along the vertical axis highlight the proportions of institutions where no death was reported. Grey line $=$ per FE institution, $\mathrm{NH}=$ nursing homes, HEP = homes for elderly people. The two size classes per FE category are based on bed capacity (mean bed capacity indicated in the graph legend with the overall death rate).

Source: AViQ.

The crude COVID-19 death rate per institution showed a slight positive spatial autocorrelation between institutions separated by less than $10 \mathrm{~km}$ (Moran's I $=0.058$, pvalue $=0.003$ ) but no departure from spatial randomness at larger distances. Hence, there was no large-scale gradient of mortality rates across Wallonia.

\subsection{Temporal dynamics of COVID-19 deaths inside and outside FE}

Mortality levels by COVID-19 are much higher in FE than outside, and the temporal dynamics of mortality also differed in the two populations. Until April 5, 2020 the majority of deaths had occurred in the OUT-FE population, while FE residents constituted the majority of cumulated deaths after April 6, 2020 (Figure 2). The slight delay in the mortality dynamics of FE residents can be quantified by the date at which $50 \%$ of the deaths reported in the observed period occurred: 3.5 days later in the FE population (April 13) than in the OUT-FE population (April 10). The wave of deaths was also more concentrated in FE, with 52 days between the dates when $5 \%$ and $95 \%$ of the total deaths were reached (i.e., between March 28 and May 19), than in the OUT-FE population (65 days between March 23 and May 28). Hence, according to mortality data, 
the outbreak in FE was delayed but then spread faster than the outbreak in the OUT-FE population.

\section{Figure 2: Cumulated number of deaths attributed to COVID-19 in Wallonia,} March - June 2020

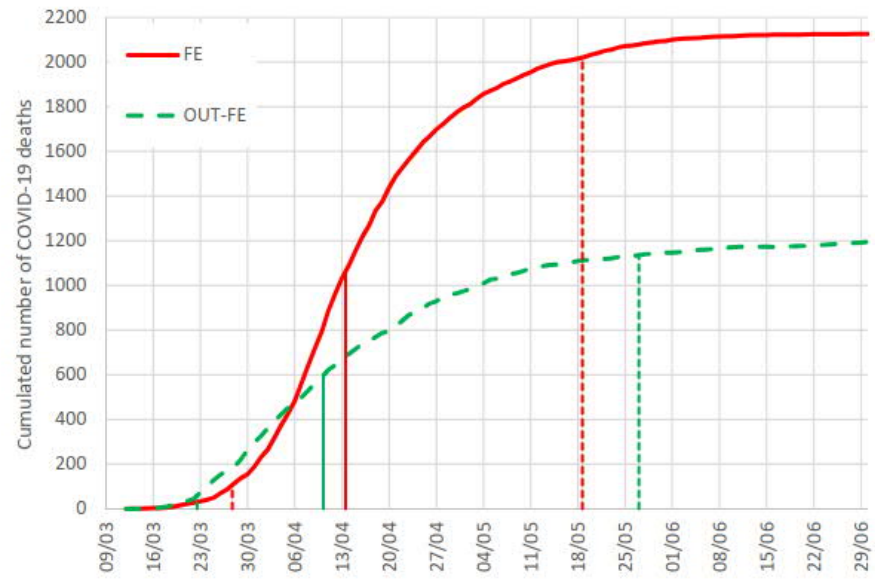

Notes: The dates at which $50 \%$ of the total deaths recorded in each population are indicated by vertical bars, while the 0.05 and 0.95 quantiles are indicated by vertical stippled lines. FE, red plain line $=$ persons living in residential facilities for elderly people, OUT-FE, green stippled line $=$ the rest of the population.

Sources: Sciensano, AViQ

\subsection{Age and sex structure effect on COVID-19 death rate}

The FE population, which constitutes $1.35 \%$ of the French-speaking part of Wallonia (3,567,294 inhabitants), has a peculiar age structure, with people aged 65 and over constituting $95 \%$ of the total population, compared to $18 \%$ in the OUT-FE population (Figure 3). Moreover, among the people aged 65 and over, the oldest (85 and over) are over-represented in FE, accounting for $60 \%$ compared to only $11 \%$ in the OUT-FE population. As a result, the mean age is 84.2 years $(\mathrm{SD}=9.6)$ in the $\mathrm{FE}$ and only 41.0 years $(\mathrm{SD}=23.2)$ outside the FE. The gender structure in the FE is also very particular, characterized by a highly biased sex ratio, with $74.6 \%$ of females $(51.1 \%$ in the overall population), a percentage increasing from $50.9 \%$ among the $60-64$ years old to $91.2 \%$ in the $\geq 100$ years old. Within the FE, the age pyramids of NH and HEP residents are very similar, the main difference being a slightly higher proportion of males 60-74 years old 
in HEP $(8.0 \%)$ compared to $\mathrm{NH}(6.3 \%)$, compensated by a lower proportion of males 80 94 years old in HEP (12.2\%) compared to NH (13.8\%).

Figure 3: Age pyramids (in \%) of the populations of the French-speaking part of Wallonia on January 1, 2020

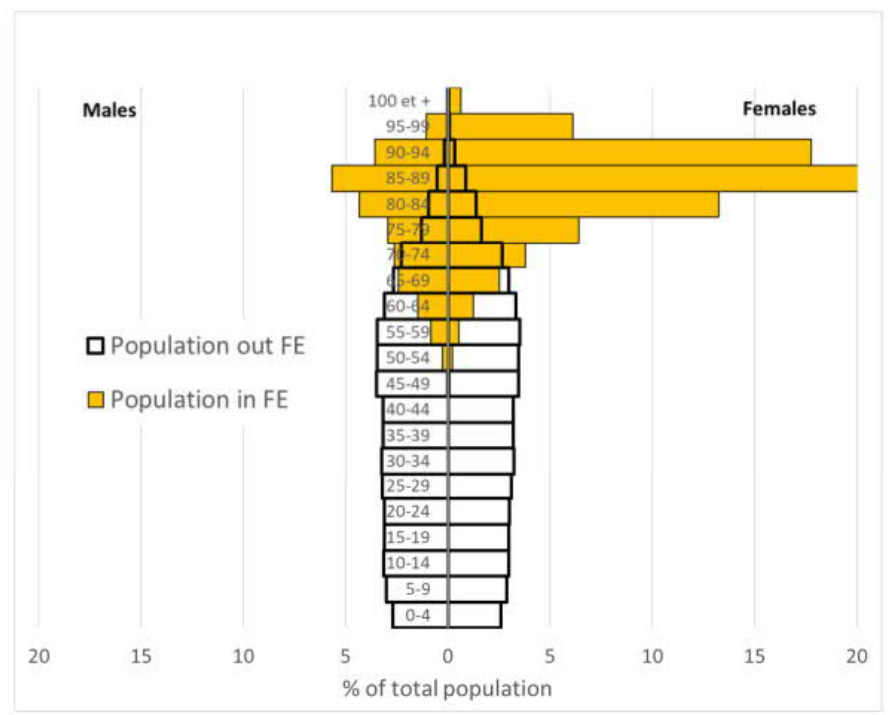

Notes: The $65+$ people made $95.1 \%$ of the FE population and $17.8 \%$ of the OUT-FE population. Orange = people living in residential facility for elderly people (FE), white = the rest of the population (OUT-FE).

Sources: StatBel, AViQ.

Given the larger number of female FE residents, especially among the oldest and more vulnerable age classes, females constitute a majority (66\%) of the COVID-19 deaths in FE. By contrast, in the OUT-FE population composed of $51 \%$ females, $40 \%$ of COVID-19 deaths were females. Overall, in Wallonia, females constituted 55\% of COVID-19 deaths despite their lower vulnerability (see below) because among the people $\geq 65$ years the proportion of females living in FE (9.2\%) is much larger than the proportion of males living in FE (3.9\%).

The ExpCDR_FE reaches 3.71\%o, which is 10.9 times higher than the CDR outside FE $(0.34 \%$ ) . Hence, the difference in age pyramids between the populations, i.e., the (age and sex) structure effect, already predicts an 11-fold higher COVID-19 death rate inside than outside the FE population $(\mathrm{CRR}=10.9)$. 


\subsection{COVID-19 death rates by sex and age group - health condition effect}

In both the FE and OUT-FE populations, COVID-19 ASDR (confirmed and suspected) closely followed the Gompertz law (i.e., exponential increase with age), but with a lower slope in FE (mortality doubling every 24 years in $\mathrm{M}$ and 18 years in F) than OUT-FE (mortality doubling every 7.5 years in $\mathrm{M}$ and 6.6 years in F) (Figure 4a). COVID-19 death rates were higher in males than in females of the same age class, by a factor of c. 2.0 among those aged 65-99 years, both inside and outside FE. As already shown with the distribution of death rates per institution (Figure 1), there was also a sharp contrast between NH and HEP populations: across all ages, COVID-19 death rates in NH (6.9\% in $\mathrm{M}, 4.1 \%$ in $\mathrm{F}$ ) were approximately $2.9(\mathrm{M})$ to $2.5(\mathrm{~F})$ times higher than in HEP $(2.4 \%$ in $\mathrm{M}, 1.7 \%$ in $\mathrm{F})$.

Figure 4: Age- and sex-specific death rates due to (a) COVID-19 during spring 2020 and (b) all causes during 2017, Wallonia

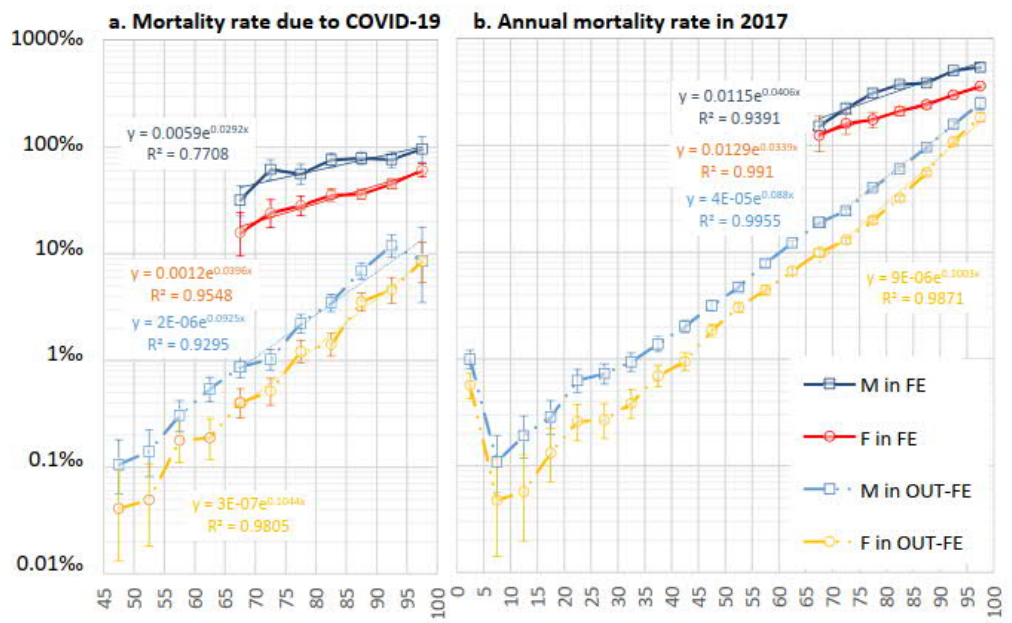

Notes: Death rates are represented on a logarithmic scale. Equations and thin straight lines represent the best-fitting exponential curves adjusted to the 65-99 age classes. Plain lines = FE, residential facilities for elderly people; stippled-dotted lines = OUT-FE, the rest of the population; squares $=$ males, circles $=$ females.

Sources: Sciensano, AViQ, StatBel.

At all ages, COVID-19 death rates were much higher in FE than outside FE (Figure $4 a)$, reflecting the health condition effect. However, the ratio of FE to OUT-FE rates tends to decrease with age: while it exceeds 30 for people under 75 , it reaches 10 for those aged 85-89 (the age group where most COVID-19 deaths are concentrated, both inside and 
outside FE), and 4 for people over 99 years. Thus, the mortality differential is most pronounced at the youngest ages.

The SMR_EF, which measures the contribution of differences in COVID-19 ASDR between FE and OUT-FE populations, i.e., the health condition effect, is 11.9 (44.2\%o / $3.71 \%$ o). Consequently, the excess mortality observed in FE results from the combination of a structure effect (x 10.9) and a health condition effect (x 11.9), their product giving the ratio between the CDR of the two populations $(10.9 \times 11.9 \approx 130)$.

\subsection{All-cause annual death rate by sex and age group - a proxy for overall health frailty}

When excluding child mortality ( $0-4$ years class), the ASDR based on 2017 mortality data also closely followed the Gompertz law in both FE and OUT-FE populations, but were overall 5 times (in FE) to 20 times (OUT-FE) higher than the COVID-19 death rates (Figure $4 \mathrm{~b}$ ). Age-specific death rates were higher in males $(\mathrm{M})$ than in females $(\mathrm{F})$ of the same age class, by a factor of c.1.6 in FE and 1.7 in OUT-FE among those aged 65-99 years. However, for the same age class, all-cause mortality rates were much higher in than outside FE and increased with age less rapidly in FE (mortality doubling every 17 years in $\mathrm{M}$ and 20 years in F) than OUT-FE (mortality doubling every 8 years in M and every 7 years in F). Therefore, while all-cause death rates were c. 10 times higher in FE than in OUT-FE for the 65-69 class, they remained only c. 2 times higher for the 95-99 class (Figure 4b), indicating that the morbidity of care home residents tends to be stronger among the youngest residents when their age is taken into account.

In 2017 the all-cause annual CDR was 266.7\% in FE compared to 7.0\%o in the OUTFE population, a 38 -fold difference. Using the ASDR observed outside FE, weighted by the size of the FE population, predicts a crude death rate of $70.7 \%$ in the FE population if it was as healthy as the OUT-FE population in the same age pyramid. Hence, the relative health frailty of the FE population compared to the OUT-FE population is RF = $266.7 / 70.7=3.77$. Across all age classes, annual all-cause death rates in $\mathrm{NH}(34.1 \%$ in $\mathrm{M}, 25.2 \%$ in F) were approximately 1.2 times higher than in $\operatorname{HEP}(27.6 \%$ in $\mathrm{M}, 20.1 \%$ in $\mathrm{F})$, reflecting the fact that $\mathrm{NH}$ institutions host a higher proportion of residents in poor health than HEP institutions. 


\subsection{Comparison of COVID-19 and annual ASDR to infer differences in contamination rates between populations}

While mortality levels by age and sex due to COVID-19 in March-June 2020 are lower than all-cause annual mortality in 2017, within each population and sex the COVID-19 ASDR is nearly proportional to the annual ASDR (Figure 4a, b). However, there are two major differences between COVID-19 and all-cause mortality. First, the gap between COVID-19 mortality and all-cause mortality is lower in care homes than in the rest of the population (Fig 4a). Second, the excess mortality in males, already present in the allcause mortality, is even higher in the case of COVID-19 mortality. In and outside FE, while the male-to-female mortality ratio for those aged $65+$ is c. 1.6 in 2017, for COVID19 mortality it exceeded 2.0.

The fact that within and outside FE the COVID-19 ASDR is nearly proportional to the all-cause ASDR suggests that the latter might be a good proxy for the average effect of comorbidity factors (or health frailty) conditioning the risk of dying from COVID-19 when one is infected. Under this assumption, we can tentatively factor out the impact of comorbidity factors using the ratio of COVID-19 to all-cause annual death rates for each age class and sex (Figure 5). Although these ratios are slightly higher for males than females in both the FE and OUT-FE populations, and a little higher before age 75 than at later ages for men in FE, the main difference is between populations: while COVID-19 death rates are around $16 \%$ (F) to $20 \%(\mathrm{M})$ of the all-cause annual death rates in FE, they reached only $4.6 \%(\mathrm{~F})$ to $5.3 \%(\mathrm{M})$ of the all-cause annual death rates outside FE. If we assume that this difference results from different levels of exposure to the SARS-CoV-2 virus causing COVID-19, FE residents would have been c. 3.4 times more contaminated than the OUT-FE population of similar age $(\mathrm{RI}=3.42$ across all age classes and sex). Moreover, average ratios for those aged 65-99 distinguishing NH from HEP populations indicate that the contamination was probably $1.6(\mathrm{HEP})$ to $3.8(\mathrm{NH})$ times higher than in the OUT-FE population, and thus that $\mathrm{NH}$ institutions would have been at least twice more contaminated than HEP institutions. 


\section{Figure 5: $\quad$ Ratio of COVID-19 to annual death rates}

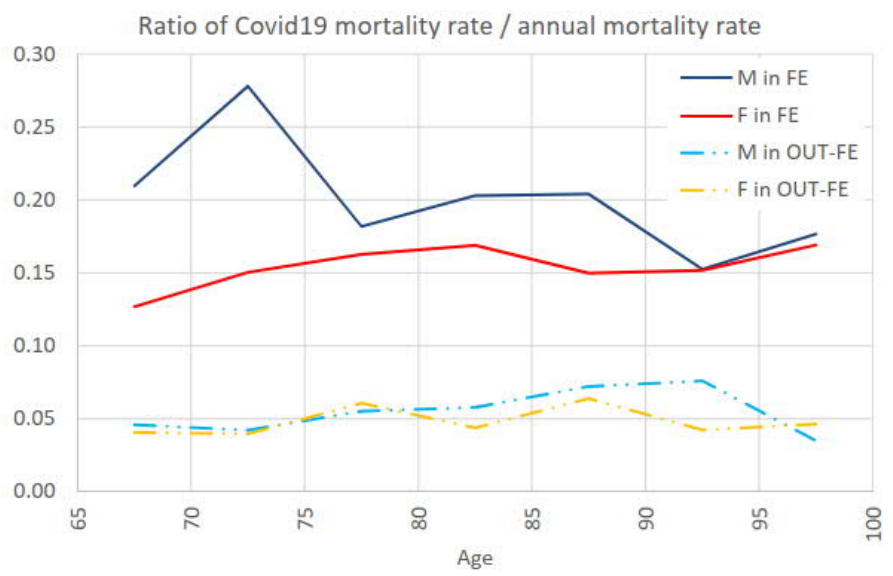

Note: blue $=$ males; red orange $=$ females; plain lines $=$ residential facilities for elderly people, FE; dotted-stippled lines $=$ the rest of the population, OUT-FE.

Sources: AViQ, Sciensano, StatBel.

\section{Discussion}

In Wallonia, care home residents constitute only $1.33 \%$ of the population but $64 \%$ of the COVID-19 deaths reported during spring 2020, so that the CDR due to COVID-19 was c. 130 times higher than for the population living outside of care homes. According to our analyses, this seemingly dramatic ratio results from the combination of three main factors. First, the peculiar age pyramid of the care home population predicts a CDR 11 times higher than that of the external population, because COVID-19 is much more deadly for older people (structure effect). Second, the low average health condition of care home residents increases their all-cause annual death rate by c. 3.8 times compared to the external population after accounting for age and sex effects, while COVID-19 mortality appears highly correlated with the presence of other comorbidity factors (health frailty effect). Finally, care home residents were probably on average 3.4 times more exposed to the SARS-CoV-2 virus than the external population of similar age (1.6 times more in HEP, 3.8 times more in $\mathrm{NH}$ ), and tended to be more exposed when living in larger institutions (higher SARS-CoV-2 prevalence). All these effects worked nearly multiplicatively to generate the high death toll in care homes. These inferences are based on a number of assumptions that merit discussion. 
Our decomposition of the excess CDR in care homes in three components is based on different ratios of CDR or ASDR to COVID-19 or to annual deaths reported inside and outside care homes (Appendix). However, some of these death rates can be biased by limited available data (see Material and Methods). In particular, the COVID-19 death rate estimated within FE could be somewhat underestimated because during the epidemic the FE did not take in new residents when places were vacated by deceased residents (Appendix). Consequently, our estimates of the excess of COVID-19 CDR in FE (130 times) and of the contamination excess in FE (3.4 times) tend to be conservative, as they might also have been somewhat underestimated. On the other hand, the structure effect (11 times) is very slightly overestimated, since proportionally COVID-19 mortality affected the FE population more and thus reduced the number of people at risk of death.

The higher mortality of COVID-19 in older people has been reported since the beginning of the epidemic (Verity et al. 2020). Our analyses using 5-year age classes allow us to better characterize this relationship, showing that the death rate doubles approximately every 6 years of age increment for people living outside of care homes, and every 20 years for care home residents, a result similar to that observed by Bauer et al. (2021) in a comparison of COVID-19 ASDR in Western Europe and the United States. This type of mortality-age relationship, known as the Gompertz law (Gompertz 1825), often fits well mortality data for a wide range of causes (Riggs 1991 for ischemic heart disease in the United States; Imaizumi 1996 for breast cancer in Japan), as well as total mortality rates at the national level (all causes confounded, Horiuchi et al. 2003; Dolejs 2015), as confirmed in our analyses of the annual mortality rate (if we exclude child mortality). What is remarkable here is that the exponents of the Gompertz law adjusted to our data were very similar for COVID-19 mortality and all-cause annual mortality (Figure 4a, b), while they diverged strongly between populations living inside and outside care homes. This strong correlation between COVID-19 and all-cause mortality rates suggests that the risk of dying from COVID-19 largely depends on the person's initial health condition, as supported by numerous clinical observations showing the strong impact of multiple comorbidity factors (Williamson et al. 2020) or of a frailty index (Hewitt et al. 2020). In contrast to our results, Bauer et al. (2021) reported a more pronounced age dependency for COVID-19 mortality than for all-cause mortality using death data at the national level, a pattern also found in Wallonia if we aggregate death data within and outside care homes (mortality doubling every 5.5 and 6.4 years of age increment for COVID-19 and all-cause mortality, respectively, results not shown). Hence, the higher COVID-19 age dependency observed at the national level probably results from the higher infections in cares homes where the prevalence of comorbidities is high, rather than from a larger impact of comorbidities on COVID-19 mortality than on all-cause mortality. 
That males have higher COVID-19 mortality than females has also been reported since the beginning of the epidemic (Verity et al. 2020; Wenham, Smith, and Morgan 2020; Williamson et al. 2020). In Wallonia the COVID-19 death rate for males was approximately double that of females of the same age, and appears higher than the male mortality excess observed for all-cause mortality rates unrelated to COVID-19 (M:F mortality ratio from 1:6 to 1:7), a result also reported in other Western countries (Bauer et al. 2021). Interestingly, this excess mortality in males was identical when considering suspected and confirmed cases of COVID-19 deaths in care homes (results not shown), suggesting that most suspected cases were correctly diagnosed. This is also supported by the very good quantitative correspondence between the daily deaths attributed to COVID19 (including suspected cases) and the daily death excess occurring in spring 2020 compared to previous years (Molenberghs et al. 2020; Wu et al. 2020). Despite their higher vulnerability, males constituted only $44.8 \%$ of COVID-19 deaths in Wallonia, a paradox resulting from the much lower proportion of males (3.9\%) than females (9.2\%) aged $\geq 65$ years old living in care homes, while the care home population has been more infected.

We tentatively interpreted the higher ratio of COVID-19 to all-cause death rates in care homes compared to the outside population as reflecting a higher contamination in care homes, due to the potentially rapid spread of the virus in such an environment (Arons et al. 2020). However, other factors may also play a role, in particular the quality of care treatment, given that a minority of care home residents who died from COVID-19 were hospitalized (28\%), whereas nearly all victims in the OUT-FE population were hospitalized. Moreover, during the peak of the COVID-19 infection wave of spring 2020, in particular from April to early May, the proportion of deaths of care home residents in hospital was lower (26\%) than just before and after (41\%), possibly because the risk of saturating hospitals' intensive care facilities influenced the decision of whether or not to hospitalize COVID-19 patients. Nevertheless, in hospitals the proportion of patients of whatever origin who died from COVID-19 was very high among those aged $\geq 80$ (39\%) compared to those aged $<60$ (4\%) (Van Beckhoven et al. 2020), so that even if a higher proportion of care home residents were hospitalized it may not have significantly changed their chance of survival, as suggested by the absence of correlation between the COVID19 death rates per care home and the proportion of deaths that occurred in hospitals (results not shown). Moreover, differences in care quality cannot explain why HEP, in which $40 \%$ of the residents who died from COVID-19 were hospitalized, were 2.4 times less affected than $\mathrm{NH}$ institutions, even after factoring out the different health conditions of their respective populations. The lower level of close contact with caregivers in HEP compared to $\mathrm{NH}$ is a more parsimonious explanation of this contrast.

We currently lack serological testing data which would allow comparison of the level of presumed infection in the different populations, but for the general population in 
Belgium, seroprevalence reached $6.0 \%$ (95\% CI 5.1 to 7.1; Herzog et al. 2020) in the week of April 20-26, 2020, a value similar to that reported in other countries with a high incidence of the virus (Eckerle and Meyer 2020). If we assume that contamination by SARS-CoV-2 has been 3.4 more prevalent in care homes than outside them, we could expect a seroprevalence of c. $20 \%$ in care homes.

Other lines of evidence of the importance of viral transmission in care homes come from (1) the temporal dynamic of deaths and (2) the distribution of COVID-19 deaths and cases per institution. First, the delayed but then rapid increase of deaths in care homes compared to the external population (Figure 2) is consistent with primary infections originating from the care home staff, or possibly visitors (explaining the delay), followed by a more rapid viral contamination within each contaminated institution due to difficulties limiting interpersonal contact and/or the lack of PPE (explaining the more rapid increase). Second, the crude COVID-19 death rate was very heterogeneous among care home institutions, with 54\% of them having no COVID-19 deaths, $17 \%$ of them losing at least $100 \%$ of their residents, and $2.6 \%$ losing between $200 \%$ and up to $340 \%$. The spatial autocorrelation of these mortality rates was weak, limited to a radius of 10 $\mathrm{km}$, possibly reflecting the impact of local clusters and/or the movement of staff between related care homes, but we lack data to test the latter hypothesis. If we assume that in the most affected institutions virtually all the residents were infected, leading to an average mortality rate of $250 \%$, a mean mortality rate of $44.2 \%$ over all institutions would correspond to a mean prevalence of $44.2 / 250 \approx 18 \%$. Hence, a different reasoning again suggests that about a fifth of care home residents were contaminated by SARS-CoV-2.

The absence of COVID-19 deaths mostly occurred in HEP (without healthcare facilities) and in small institutions, while high mortality rates mostly occurred in $\mathrm{NH}$ and tended to increase with the bed capacity of the institution (Figure 1). Hence, the risk of the SARS-SoV-2 virus entering and spreading through a care home clearly increased with the healthcare staff presence and size of the institution, as expected from the potential number of interpersonal contacts during a period when PPE and possibly adequate staffing for such an epidemic were lacking. It is worth noting that the nearly two-fold lower mortality rate observed in medium-sized HEP $(2.1 \%)$ compared to $\mathrm{NH}(4.0 \%)$ is very consistent with the distribution of positive COVID-19 tests performed from the end of April to May 2020 (Hoxha et al. 2020). In institutions where at least 50\% of the residents and staff were tested, positive tests reached $2.5 \%$ for residents and $1.6 \%$ for staff in HEP (7,402 persons tested in 100 institutions), against $5.9 \%$ for residents and $4.2 \%$ for staff in $\mathrm{NH}(60,667$ persons tested in 370 institutions; unpublished results). The importance of transmission by staff was also highlighted in six care homes in London, where SARS-CoV-2 genome sequencing showed that there were often multiple introductions of the virus per institution and that staff working across different care homes had a 3-fold higher risk of being contaminated than staff working in single care 
homes (Ladhani et al. 2020). Conversely, in France, 17 nursing homes where the staff decided to self-confine voluntarily with the residents at the beginning of the pandemic recorded 4 to 8 times less cases and deaths than the national average (Belmin et al. 2020).

\section{Conclusion}

In Wallonia in Belgium, as in other countries where care homes have been heavily impacted by COVID-19, the epidemic showed distinct dynamics in and outside care homes (see Humblet 2020 for Belgium; Logar 2020 for Italy; Rada 2020 for Spain). Care homes are a very specific context because of the particular vulnerability of their population and the difficulty of containing SARS-CoV-2 transmission once it has infected an institution. Given the high death toll in care homes in many Western countries, special attention should be paid to understanding how to avoid primary infection and how to limit contamination of residents and staff. Our global analyses of death rates reveal that institution size and the nursing services provided (higher in $\mathrm{NH}$ than in HEP) were important factors determining the relative death toll. Nevertheless, our analyses do not reveal why nearly half of the institutions had no deaths while nearly one in six lost at least a tenth of their residents. These contrasts may result from (1) the stochastic nature of a primary infection (as suggested by the institution size effect), (2) differences between institutions in the mean health status of residents (e.g., HEP versus $\mathrm{NH}$ ), or (3) differences in the organisation and/or specific measures taken by each institution in response to the COVID-19 epidemic. Further research investigating the history of infections within representative institutions could clarify these questions. A comparison of serologic tests conducted inside and outside care homes would also be helpful in confirming our interpretation that SARS-CoV-2 was much more prevalent in care homes than outside them. Finally, we recommend that epidemiological models integrate care home populations as a specific entity in their forecasts.

\section{Acknowledgments}

We thank Patrick Lusyne from StatBel for helping us obtain the adequate population and annual deaths data, and for interesting discussions on the interpretation of our results. We also warmly thank the staff of the hospitals and care homes for their conscientious reporting of COVID-19 deaths despite the difficult times. We are grateful to two anonymous reviewers for their constructive comments. OJH, SD and TE are supported by the Fonds de la Recherche Scientifique (F.R.S. - FNRS, Belgium). 


\section{References}

Armitage, R. and Nellums, L.B. (2020). COVID-19 and the consequences of isolating the elderly. The Lancet Public Health 5(5): e256. doi:10.1016/S2468-2667(20) 30061-X.

Arons, M.M., Hatfield, K.M., Reddy, S.C., Kimball, A., James, A., Jacobs, J.R., Taylor, J., Spicer, K., Bardossy, A.C., Oakley, L.P., Tanwar, S., Dyal, J.W., Harney, J., Chisty, Z., Bell, J.M., Methner, M., Paul, P., Carlson, C.M., McLaughlin, H.P., and Public Health-Seattle and King County and CDC COVID-19 Investigation Team (2020). Presymptomatic SARS-CoV-2 infections and transmission in a skilled nursing facility. The New England Journal of Medicine 382(22): 20812090. doi:10.1056/NEJMoa2008457.

Ars, P., Dal, L., and Poulain, M. (1988). Comment appréhender le problème statistique des petits nombres en démographie? In: Les migrations internationales (Actes du colloque de l'AIDELF de Calabre, 1986): 156-170

Bauer, P., Brugger, J., König, F., and Posch, M. (2021). An international comparison of age and sex dependency of COVID-19 deaths in 2020: A descriptive analysis. Scientific Reports 11(1): 19143. doi:10.1038/s41598-021-97711-8.

Belmin, J., Um-Din, N., Donadio, C., Magri, M., Nghiem, Q.D., Oquendo, B., Pariel, S., and Lafuente-Lafuente, C. (2020). Coronavirus disease 2019 outcomes in French nursing homes that implemented staff confinement with residents. JAMA Network Open 3(8): e2017533-e2017533. doi:10.1001/jamanetworkopen.2020.17533.

Bustos Sierra, N., Bossuyt, N., Braeye, T., Leroy, M., Moyersoen, I., Peeters, I., Scohy, A., Van der Heyden, J., Van Oyen, H., and Renard, F. (2020). All-cause mortality supports the COVID-19 mortality in Belgium and comparison with major fatal events of the last century. Archives of Public Health 78(1): 117. doi:10.1186/ s13690-020-00496-x.

Comas-Herrera, A., Ashcroft, E., and Lorenz-Dant, K. (2020). International examples of measures to prevent and manage COVID-19 outbreaks in residential care and nursing home settings. (Report in LTCcovid.org, International Long-Term Care Policy Network, CPEC-LSE, 11 May 2020). https://tccovid.org/wp-content/ uploads/2020/05/International-measures-to-prevent-and-manage-COVID19infections-in-care-homes-2-May-1.pdf. 
Comas-Herrera, A., Zalakaín, J., Litwin, C., Hsu, A.T., Lemmon, E., Henderson, D., and Fernández, J.-L. (2020). Mortality associated with COVID-19 outbreaks in care homes: early international evidence. (Article in LTCcovid.org, International Long-Term Care Policy Network, CPEC-LSE, 26 June 2020). https://ltccovid.org/wp-content/uploads/2020/06/Mortality-associated-withCOVID-among-people-who-use-long-term-care-26-June-1.pdf.

Dolejs, J. (2015). Age trajectories of mortality from all diseases in five countries of Central Europe during the last decades. Biodemography and Social Biology 61(1): 40-64. doi:10.1080/19485565.2014.936999.

Dudel, C., Riffe, T., Acosta, E., van Raalte, A., Strozza, C., and Myrskylä, M. (2020). Monitoring trends and differences in COVID-19 case-fatality rates using decomposition methods: Contributions of age structure and age-specific fatality. PLOS ONE 15(9): e0238904. doi:10.1371/journal.pone.0238904.

Eckerle, I. and Meyer, B. (2020). SARS-CoV-2 seroprevalence in COVID-19 hotspots. The Lancet 396(10250): 514-515. doi:10.1016/S0140-6736(20)31482-3.

ECDC Public Health Emergency Team, Danis, K., Fonteneau, L., Georges, S., Daniau, C., Bernard-Stoecklin, S., Domegan, L., O’Donnell, J., Hauge, S.H., Dequeker, S., Vandael, E., Van der Heyden, J., Renard, F., Sierra, N.B., Ricchizzi, E., Schweickert, B., Schmidt, N., Abu Sin, M., Eckmanns, T., Paiva, J.-A., Schneider, E. (2020). High impact of COVID-19 in long-term care facilities, suggestion for monitoring in the EU/EEA, May 2020. Eurosurveillance 25(22): pii=2000956. doi:10.2807/1560-7917.ES.2020.25.22.2000956.

Einiö, E., Guilbault, C., Martikainen, P., and Poulain, M. (2012). Gender differences in care home use among older Finns and Belgians. Population 67(1): 71-95. doi:10.3917/pope.1201.0071.

Falconer, M. and O'Neill, D. (2007). Profiling disability within nursing homes: a censusbased approach. Age and Ageing 36(2): 209-213. doi:10.1093/ageing/afl185.

Fisman, D.N., Bogoch, I., Lapointe-Shaw, L., McCready, J., and Tuite, A.R. (2020). Risk factors associated with mortality among residents with Coronavirus disease 2019 (COVID-19) in long-term care facilities in Ontario, Canada. JAMA Network Open 3(7): e2015957-e2015957. doi:10.1001/jamanetworkopen.2020.15957.

Gompertz, B. (1825). XXIV. On the nature of the function expressive of the law of human mortality, and on a new mode of determining the value of life contingencies. In a letter to Francis Baily, Esq. F.R.S.\&c. Philosophical Transactions of the Royal Society of London 115: 513-583. doi:10.1098/rstl.1825.0026. 
Herm, A., Poulain, M., and Anson, J. (2014). Excess mortality risks in institutions: The influence of health and disability status. In: Anson, J. and Luy, M. (eds.). Mortality in an international perspective. Cham: Springer: 245-263. doi:10.1007/978-3319-03029-6_10.

Herzog, S., Bie, J.D., Abrams, S., Wouters, I., Ekinci, E., Patteet, L., Coppens, A., Spiegeleer, S.D., Beutels, P., Damme, P.V., Hens, N., and Theeten, H. (2020). Seroprevalence of $\mathrm{IgG}$ antibodies against SARS coronavirus 2 in Belgium: A prospective cross-sectional study of residual samples. MedRxiv doi:10.1101/ 2020.06.08.20125179.

Hewitt, J., Carter, B., Vilches-Moraga, A., Quinn, T.J., Braude, P., Verduri, A., Pearce, L., Stechman, M., Short, R., Price, A., Collins, J.T., Bruce, E., Einarsson, A., Rickard, F., Mitchell, E., Holloway, M., Hesford, J., Barlow-Pay, F., Clini, E., and Guaraldi, G. (2020). The effect of frailty on survival in patients with COVID-19 (COPE): A multicentre, European, observational cohort study. The Lancet Public Health 5(8): e444-e451. doi:10.1016/S2468-2667(20)30146-8.

Horiuchi, S., Finch, C.E., Meslé, F., and Vallin, J. (2003). Differential patterns of agerelated mortality increase in middle age and old age. The Journals of Gerontology Series A: Biological Sciences and Medical Sciences 58(6): B495-B507. doi:10.1093/gerona/58.6.B495.

Hoxha, A., Wyndham-Thomas, C., Klamer, S., Dubourg, D., Vermeulen, M., Hammami, N., and Cornelissen, L. (2020). Asymptomatic SARS-CoV-2 infection in Belgian long-term care facilities. The Lancet Infectious Diseases 21(4): e67. doi:10.1016/ S1473-3099(20)30560-0.

Humblet, P. (2020). Inégalités sociales de santé: Tout a changé? Politique 112: 99-102

Imaizumi, Y. (1996). Longitudinal analysis of mortality from breast cancer in Japan, 1950-1993: Fitting Gompertz and Weibull functions. Mechanisms of Ageing and Development 88(3): 169-183. doi:10.1016/0047-6374(96)01735-6.

Keiding, N. and Clayton, D. (2014). Standardization and control for confounding in observational studies: A historical perspective. Statistical Science 29(4): 529-558. doi:10.1214/13-STS453.

Ladhani, S.N., Chow, J.Y., Janarthanan, R., Fok, J., Crawley-Boevey, E., Vusirikala, A., Fernandez, E., Perez, M.S., Tang, S., Dun-Campbell, K., Evans, E.W.-, Bell, A., Patel, B., Amin-Chowdhury, Z., Aiano, F., Paranthaman, K., Ma, T., SaavedraCampos, M., Myers, R., and Ramsay, M.E. (2020). Increased risk of SARS-CoV2 infection in staff working across different care homes: Enhanced CoVID-19 
outbreak investigations in London care homes. The Journal of Infection 81(4): 621-624. doi:10.1016/j.jinf.2020.07.027.

Laferrère, A., Van den Heede, A., Van den Bosch, K., and Geerts, J. (2013). 22 entry into institutional care: predictors and alternatives. In: Börsch-Supan, A., Brandt, M., Litwin, H., and Weber, G. (eds.). Active ageing and solidarity between generations in Europe: First results from SHARE after the economic crisis. Berlin: DeGruyter: 253-264. doi:10.1515/9783110295467.253.

Logar, S. (2020). Care home facilities as new COVID-19 hotspots: Lombardy Region (Italy) case study. Archives of Gerontology and Geriatrics 89(SI COVID-19): 104087. doi:10.1016/j.archger.2020.104087.

Medford, A. and Trias-Llimós, S. (2020). Population age structure only partially explains the large number of COVID-19 deaths at the oldest ages. Demographic Research 43(19): 533-544. doi:10.4054/DemRes.2020.43.19.

Miettinen, O.S. (1972). Components of the crude risk ratio. American Journal of Epidemiology 96(2): 168-172. doi:10.1093/oxfordjournals.aje.a121443.

Molenberghs, G., Faes, C., Aerts, J., Theeten, H., Devleesschauwer, B., Bustos Sierra, N., Braeye, T., Renard, F., Herzog, S., Lusyne, P., Van der Heyden, J., Van Oyen, H., Van Damme, P., and Hens, N. (2020). Belgian COVID-19 mortality, excess deaths, number of deaths per million, and infection fatality rates (8 March-9 May 2020) [Preprint]. MedRxiv. doi:10.1101/2020.06.20.20136234.

Nepomuceno, M.R., Acosta, E., Alburez-Gutierrez, D., Aburto, J.M., Gagnon, A., and Turra, C.M. (2020). Besides population age structure, health and other demographic factors can contribute to understanding the COVID-19 burden. Proceedings of the National Academy of Sciences 117(25): 13881-13883. doi:10.1073/pnas.2008760117.

Peeters, I., Vermeulen, M., Bustos Sierra, N., Renard, F., Van der Heyden, J., Scohy, A., Braeye, T., Bossuyt, N., Haarhuis, F., Proesmans, K., Vernemmen, C., and Vanhaverbeke, M. (2021). Surveillance of COVID-19 mortality in Belgium, epidemiology and methodology during 1st and 2nd wave (March 2020-14 February 2021). Brussels: Sciensano. https://covid-19.sciensano.be/sites/ default/files/Covid19/COVID-19_THEMATIC\%20REPORT_SURVEILL ANCE\%20OF\%20COVID-19\%20MORTALITY\%20IN\%20BELGIUM_2.pdf.

Petretto, D.R. and Pili, R. (2020). Ageing and COVID-19: What is the role for elderly people? Geriatrics 5(2): 25. doi:10.3390/geriatrics5020025. 
Quigley, D.D., Dick, A., Agarwal, M., Jones, K.M., Mody, L., and Stone, P.W. (2020). COVID-19 preparedness in nursing homes in the midst of the pandemic. Journal of the American Geriatrics Society 68(6): 1164-1166. doi:10.1111/jgs.16520.

Rada, A.G. (2020). Covid-19: The precarious position of Spain's nursing homes. BMJ 369: m1586. doi:10.1136/bmj.m1586.

Ricklefs, R.E. and Scheuerlein, A. (2002). Biological implications of the Weibull and Gompertz models of aging. Journals of Gerontology - Series A Biological Sciences and Medical Sciences 57(2): B69-B76. doi:10.1093/gerona/57.2.B69.

Riggs, J.E. (1991). Longitudinal Gompertzian analysis of ischemic heart disease mortality in the US, 1962-1986: A method of demonstrating the deterministic dynamics describing its decline. Mechanisms of Ageing and Development 57(1): 1-14. doi:10.1016/0047-6374(91)90020-Z.

Sciensano (2020). COVID-19 Bulletin Epidémiologique Hebdomadaire du 19 juin 2020. Brussels: Sciensano. https://covid-19.sciensano.be/sites/default/files/Covid19/ COVID-19_Weekly\%20report_20200619\%20-\%20FR.pdf.

Szczerbińska, K. (2020). Could we have done better with COVID-19 in nursing homes? European Geriatric Medicine 11: 639-643. doi:10.1007/s41999-020-00362-7.

Trabucchi, M. and De Leo, D. (2020). Nursing homes or besieged castles: COVID-19 in Northern Italy. The Lancet Psychiatry 7(5): 387-388. doi:10.1016/S2215-0366 (20)30149-8.

Van Beckhoven, D., Duysburgh, E., Montourcy, M., De Rouck, M., Vilain, A., Catteau, L., Deblonde, J., Wyndham-Thomas, C., and Van Goethem, N. (2020). Points clés de la surveillance des patients hospitalisés atteints d'une infection COVID-19 confirmée: Résultats jusqu'au 14 juin 2020. Brussels: Sciensano. Numéro de dépôt légal: D/2020/14.440/65. https://covid-19.sciensano.be/sites/default/ files/Covid19/COVID-19_THEMATIC\%20REPORT_COVID-19\%20HOSPIT ALISED\%20PATIENTS_FR.pdf.

Verbeek, H., Gerritsen, D.L., Backhaus, R., de Boer, B.S., Koopmans, R.T., and Hamers, J.P. (2020). Allowing visitors back in the nursing home during the COVID-19 crisis: A Dutch national study into first experiences and impact on well-being. Journal of the American Medical Directors Association 21(7): 900-904. doi:10.1016/j.jamda.2020.06.020.

Verity, R., Okell, L.C., Dorigatti, I., Winskill, P., Whittaker, C., Imai, N., CuomoDannenburg, G., Thompson, H., Walker, P.G.T., Fu, H., Dighe, A., Griffin, J.T., Baguelin, M., Bhatia, S., Boonyasiri, A., Cori, A., Cucunubá, Z., FitzJohn, R., 
Gaythorpe, K., and Ferguson, N.M. (2020). Estimates of the severity of coronavirus disease 2019: A model-based analysis. The Lancet Infectious Diseases 20(6): 669-677. doi:10.1016/S1473-3099(20)30243-7.

Wenham, C., Smith, J., and Morgan, R. (2020). COVID-19: The gendered impacts of the outbreak. The Lancet 395(10227): 846-848. doi:10.1016/S0140-6736(20)305262.

Williamson, E.J., Walker, A.J., Bhaskaran, K., Bacon, S., Bates, C., Morton, C.E., Curtis, H.J., Mehrkar, A., Evans, D., Inglesby, P., Cockburn, J., McDonald, H.I., MacKenna, B., Tomlinson, L., Douglas, I.J., Rentsch, C.T., Mathur, R., Wong, A. Y.S., Grieve, R., and Goldacre, B. (2020). Factors associated with COVID-19related death using OpenSAFELY. Nature 584(7821): 430-436. doi:10.1038/ s41586-020-2521-4.

Wu, J., McCann, A., Katz, J., and Peltier, E. (2020). 153,000 missing deaths: Tracking the true toll of the Coronavirus outbreak. The New York Times. Retrieved 22 July 2020, from https://www.nytimes.com/interactive/2020/04/21/world/coronavirusmissing-deaths.html. 


\section{Appendix}

\section{A-1 Decomposing the excess of crude mortality rate observed in facilities for the elderly (FE).}

Notations for a specific 5-year age class (a) and sex $(s)$ :

cDi(a,s) number of COVID-19 deaths in FE (between March 13 and June 30, 2020)

$c D o(a, s)$ number of COVID-19 deaths outside FE (between March 13 and June 30, 2020)

$a D i(a, s)$ annual number of deaths (during 2017) in FE

$a D o(a, s)$ annual number of deaths (during 2017) outside FE

$c P i(a, s)$ population size in FE on $01 / 01 / 2020$

cPo $(a, s)$ population size outside FE on $01 / 01 / 2020$

$a P i(a, s)$ population size in $\mathrm{FE}$ on $01 / 01 / 2018$

$a P o(a, s)$ population size outside FE on 01/01/2018

Crude death rates $(C D R)$ due to COVID-19 $(c C D R)$ or all causes within a year $(a C D R)$, within FE $(C D R i)$ and outside FE $(C D R o)$ :

$$
\begin{array}{r}
c C D R i=\frac{\sum_{a, s} c \operatorname{Di}(a, s)}{\sum_{a, s} c \operatorname{Pi}(a, s)} \\
c C D R o=\frac{\sum_{a, s} c D o(a, s)}{\sum_{a, s} c \operatorname{Po}(a, s)} \\
a C D R i=\frac{\sum_{a, s} a D i(a, s)}{\sum_{a, s} a \operatorname{Pi}(a, s)} \\
\text { aCDRo }=\frac{\sum_{a, s} a D o(a, s)}{\sum_{a, s} a \operatorname{Po}(a, s)}
\end{array}
$$

Sex- (s) and age- (a) specific death rates (ASDR) due to COVID-19 (cASDR) or all causes within a year $(a A S D R)$, within FE (ASDRi) and outside FE (ASDRo):

$$
\begin{aligned}
\text { cASDRi }(a, s) & =\frac{c D i(a, s)}{c P i(a, s)} \\
c A S D R o(a, s) & =\frac{c D o(a, s)}{c \operatorname{PPo}(a, s)} \\
\operatorname{aASDRi}(a, s) & =\frac{a \operatorname{Di}(a, s)}{a \operatorname{aPi}(a, s)}
\end{aligned}
$$




$$
\operatorname{aASDRo}(a, s)=\frac{a D o(a, s)}{\operatorname{aPo}(a, s)}
$$

Expected crude death rates within FE due to COVID-19 (cExpCDR_FE) or all causes within a year $\left(a \operatorname{Exp} C D R_{-} F E\right)$ when sex- and age-specific death rates observed outside FE $(\operatorname{ASDRo}(a, s))$ are applied to the age pyramid observed within FE:

$$
\begin{aligned}
& c \operatorname{ExpCDR\_ FE}=\frac{\sum_{a, s}(\operatorname{cASDRo}(a, s) * c P i(a, s))}{\sum_{a, s} c \operatorname{Pi}(a, s)} \\
& a E x p C D R_{-} F E=\frac{\sum_{a, s}(a A S D R o(a, s) * a P i(a, s))}{\sum_{a, s} a \operatorname{PP}(a, s)}
\end{aligned}
$$

The confounding risk ratio $(C R R)$ measures how the age pyramid of FE is expected to cause an excess of COVID-19 deaths in FE compared to outside FE (structure effect):

$$
C R R=\frac{c \operatorname{ExpCDR\_ FE}}{c C D R o}
$$

The indirect Standardized Mortality Ratio in FE ( $\left.S M R \_F E\right)$ describes the excess COVID-19 death rate in FE compared to that expected based on the deaths rates observed outside FE after accounting for the differences in these populations' age pyramids:

$$
S M R_{-} F E=\frac{c C D R i}{c E x p C D R_{-} F E}
$$

We assume that a $S M R \_F E>1$ can result from higher frailty and/or from a higher COVID-19 infection rate of the FE population compared to the population outside FE. The relative frailty of the FE population $(R F)$ is estimated similarly to the SMR_FE but using the annual all-causes death rate:

$$
R F=\frac{a C D R i}{a E x p C D R \_F E}
$$

As we noticed that the ratios of COVID-19 to annual specific death rates, $c A S D R(a, s): a A S D R(a, s)$, were relatively independent of age and sex, both inside and outside FE (Figure 5), we tentatively quantified the relative COVID-19 infection $(R I)$ of the FE population with respect to the outside population by:

$$
R I=\frac{c C D R i}{a C D R i} / \frac{C C D R o}{a C D R o}
$$


The excess of crude death rates observed in FE compared to that outside FE (cCDRi/cCDRo) tends to approach the product CCR.RF.RI, the relationship being mathematically exact only if the age pyramids are identical inside and outside FE (which is obviously not the case). Hence, $C C R, R F$, and $R C$ combine near-multiplicatively to give the ratio $c C D R i$ :cCDRo.

\section{A-2 Interpretation of death rates}

Computing classical demographic mortality rates in FE is complicated due to the high turnover of people and the availability of death data for only a single year. However, with an increase in overall bed capacity of only $0.6 \%$ between 2017 and 2018 and the fact that around $98.3 \%$ of beds were occupied, we can consider the FE population stable during a single year. Therefore, while the computed annual mortality rates overestimate the probability that an FE resident dies within a year, they can be interpreted as 365 times the probability that a FE resident dies within a day. They are thus proportional to the instantaneous rate of mortality (the so-called force of mortality) and as such represent an adequate measure of the average health status of a particular age and sex category.

The situation is different for COVID-19 mortality rates because once the epidemic started in March 2020, FE institutions usually did not admit new residents to fill their free beds until mid-June. We also ignore how many residents left institutions to be with their family. Hence, COVID-19 death rates in FE are likely somewhat underestimated. 